\title{
Tratamento de lesão por mordedura de animal - Relato de caso clínico
}

\author{
Treatment of animal bite injury - Clinical case report \\ Tratamiento de la lesión por mordedura de animal: Informe de un caso clínico
}

Stéfani Caroline Ferriolli

ORCID: https://orcid.org/0000-0003-0019-6026 Universidade Estadual Paulista, Brasil E-mail: stefani.ferriolli@outlook.com

Ana Maira Pereira Baggio

ORCID: https://orcid.org/0000-0002-9474-5091 Universidade Estadual Paulista, Brasil E-mail: anamairabaggio@gmail.com Izabela Fornazari Delamura

ORCID: https://orcid.org/0000-0001-8046-489X Universidade Estadual Paulista, Brasil

E-mail: izabela.delamura@unesp.br

Jaqueline Hassumi Suemi

ORCID: https://orcid.org/0000-0002-5214-1214 Universidade Estadual Paulista, Brasil E-mail: jaquelinehassumi@hotmail.com

Henrique Hadad

ORCID: https://orcid.org/0000-0001-64463643 Universidade Estadual Paulista, Brasil

E-mail: henriquehadad@gmail.com

Jonathas Eduardo Virgilio Piassi

ORCID: https://orcid.org/0000-0002-9241-3456 Universidade Estadual Paulista, Brasil E-mail: j.piassi@unesp.br

Leonardo Perez Faverani

ORCID: https://orcid.org/0000-0003-2249-3048 Universidade Estadual Paulista, Brasil E-mail: leonardo.faverani@unesp.br Osvaldo Magro Filho

ORCID: https://orcid.org/0000-0002-9821-2479 Universidade Estadual Paulista, Brasil E-mail: osvaldo.magro@unesp.br

Idelmo Rangel Garcia Junior

ORCID: https://orcid.org/0000-0001-8892-781X Universidade Estadual Paulista, Brasil E-mail: irgcirurgia@gmail.com

Alessandra Marcondes Aranega ORCID: https://orcid.org/0000-0001-5856-7972 Universidade Estadual Paulista, Brasil E-mail: alessandra.aranega@unesp.br

Daniela Ponzoni

ORCID: https://orcid.org/0000-0001-5928-0914 Universidade Estadual Paulista, Brasil E-mail: Daniela.ponzoni@unesp.br

Ana Paula Farnezi Bassi

ORCID: https://orcid.org/0000-0002-0031-4953 Universidade Estadual Paulista, Brasil E-mail: ana.bassi@unesp.br

\begin{abstract}
Resumo
Lesões em face, acometidas por animais domésticos, estão entre os tipos mais comuns de traumatismos, particularmente em crianças. Os ferimentos causados nessa região, podem gerar uma série de morbidades como perdas importantes de tecidos, infecções, óbito em casos mais severos, além de cicatrizes irreversíveis quando a ferida é passível de reparo. Portanto, os cuidados pós trauma devem ter como finalidade, à minimização da cicatrização, além da funcionalidade, buscando o máximo de estética possível. Uma avaliação clínica inicial minuciosa é primordial, e, a partir dela, a definição do tratamento mais adequado para o caso específico é essencial para a diminuição do risco de infecções mais graves e, consequentemente, o sucesso do tratamento desses pacientes. Este trabalho tem por objetivo relatar um caso clínico de criança vítima de mordedura animal com presença de laceração
\end{abstract}


extensa na região frontal, atendida pelo serviço de Cirurgia e Traumatologia Bucomaxilofacial da FOA Unesp, tratada por reparo primário e antibioticoterapia profilática, em que o resultado do tratamento foi considerado satisfatório com ausência de infecção.

Palavras-chave: Traumatismos faciais; Traumatismo múltiplo; Animais domésticos.

\begin{abstract}
Lesions on the face, affected by domestic animals, are among the most common types of trauma, particularly in children. The injuries caused in this region, can generate a series of morbidities such as important tissue loss, infections, death in more severe cases, in addition to irreversible scars when the wound is liable to repair, therefore, post trauma care should aim, at minimization of healing, in addition to functionality, seeking as much aesthetics as possible. A thorough initial clinical evaluation is essential, and, from there, the definition of the most appropriate treatment for the specific case is essential to reduce the risk of more serious infections and, consequently, the successful treatment of these patients. This paper aims to report a clinical case of a child victim of animal bite with the presence of extensive laceration in the frontal region, attended by the FOA Unesp Oral and Traumatology Maxillofacial Surgery service, treated by primary repair and prophylactic antibiotic therapy, in which the result of the treatment was considered satisfactory with no infection.
\end{abstract}

Keywords: Facial trauma; Multiple trauma; Domestic animals.

\begin{abstract}
Resumen
Las lesiones en la cara, afectadas por animales domésticos, se encuentran entre los tipos de traumatismos más comunes, especialmente en los niños. Las lesiones ocasionadas en esta región pueden generar una serie de morbilidades como pérdida importante de tejido, infecciones, muerte en casos más severos, además de cicatrices irreversibles cuando la herida es susceptible de reparación, por lo que la atención postraumática debe apuntar a la minimización de curativo, además de funcionalidad, buscando la mayor estética posible. Una evaluación clínica inicial minuciosa es fundamental y, a partir de ahí, la definición del tratamiento más adecuado para el caso concreto es fundamental para reducir el riesgo de infecciones más graves $\mathrm{y}$, en consecuencia, el tratamiento exitoso de estos pacientes. El presente trabajo tiene como objetivo reportar un caso clínico de un niño víctima de mordedura de animal con presencia de laceración extensa en la región frontal, atendido por el servicio de Cirugía Oral y Traumatologia Maxilofacial de la FOA Unesp, tratado mediante reparación primaria y antibioticoterapia profiláctica, en el que el resultado del tratamiento se consideró satisfactorio sin infección.
\end{abstract}

Palabras clave: Trauma facial; Trauma múltiple; Animales domésticos.

\title{
1. Introdução
}

Feridas causadas por mordedura de animais são comumente vistas em emergências hospitalares, correspondendo a aproximadamente 1\% dos atendimentos (Santos et al.,2007). Essas injúrias, com prevalência de 15\% em face, apresentam-se desde abrasões superficiais até ferimentos profundos. Crianças são as vítimas mais comuns das mordeduras caninas e, na maioria dos casos, os ataques envolvem cães conhecidos ou da própria família. Até $80 \%$ das mordeduras caninas em crianças ocorrem na cabeça e pescoço, enquanto essa região é afetada nos adultos em menos de 10\% dos casos (Macedo et al.,2006). O risco de infecção após a mordedura é determinado pelos cuidados locais, localização da lesão, uma boa anamnese, tipo de lesão e animal agressor. O momento ideal para abordagem do ferimento e a avaliação da necessidade de profilaxia antibiótica, visto o risco potencial de infecção desses ferimentos, ainda continuam discussões controversas na literatura, embora cada vez mais se defenda a abordagem por fechamento primário de ferimentos não infectados, ao invés do reparo tardio, e emprego de medicação anti-microbiana em situações específicas a serem avaliadas ao exame clínico (Porto et al.,2013). Os autores apresentam um caso de mordedura de animal (cão) atendido pelo Serviço de Cirurgia e Traumatologia Buco-Maxilo-Facial da Faculdade de odontologia de Araçatuba.

\section{Metodologia}

Este estudo trata-se de um relato de caso de forma qualitativa e descritiva (Pereira et al., 2018), trazendo dados relevantes da literatura e discute o aspecto clínico junto a abordagens terapêuticas adequadas para lesões em face acometidas por animais, correlacionada ao caso clínico apresentado de um paciente do sexo feminino, de 8 anos de idade, submetida a reparação tecidual após mordida de animal de estimação. O estudo foi aprovado pelo Comitê de Ética em Pesquisa e o Termo 
de Consentimento Livre e Esclarecido foi assinado pelos responsáveis do paciente por este ser de menor, seguindo todos os protocolos sob o mesmo Termo de Consentimento Livre e Esclarecido, respeitando os princípios éticos. (Pereira et al., 2018; Estrela et al., 2018).

\section{Caso Clínico}

Paciente com 8 anos de idade, gênero feminino, melanoderma, foi encaminhada ao serviço de Cirurgia Bucomaxilofacial pelo pronto socorro municipal devido a um acidente provocado por mordida do cachorro de estimação com presença de laceração extensa na região temporofrontonasal (figura1A). A mesma chegou com algumas suturas feitas somente para contenção da hemorragia. Após o exame físico notou-se presença de ferimento pediculado contendo epiderme, tecido subepitelial e tecido adiposo (Figura 1 B).

Figura 1. A. Laceração extensa na região temporofrontonasal causado por mordedura de cão. B. Ferimento pediculado contendo epiderme, tecido sub-epitelial e tecido adiposo.

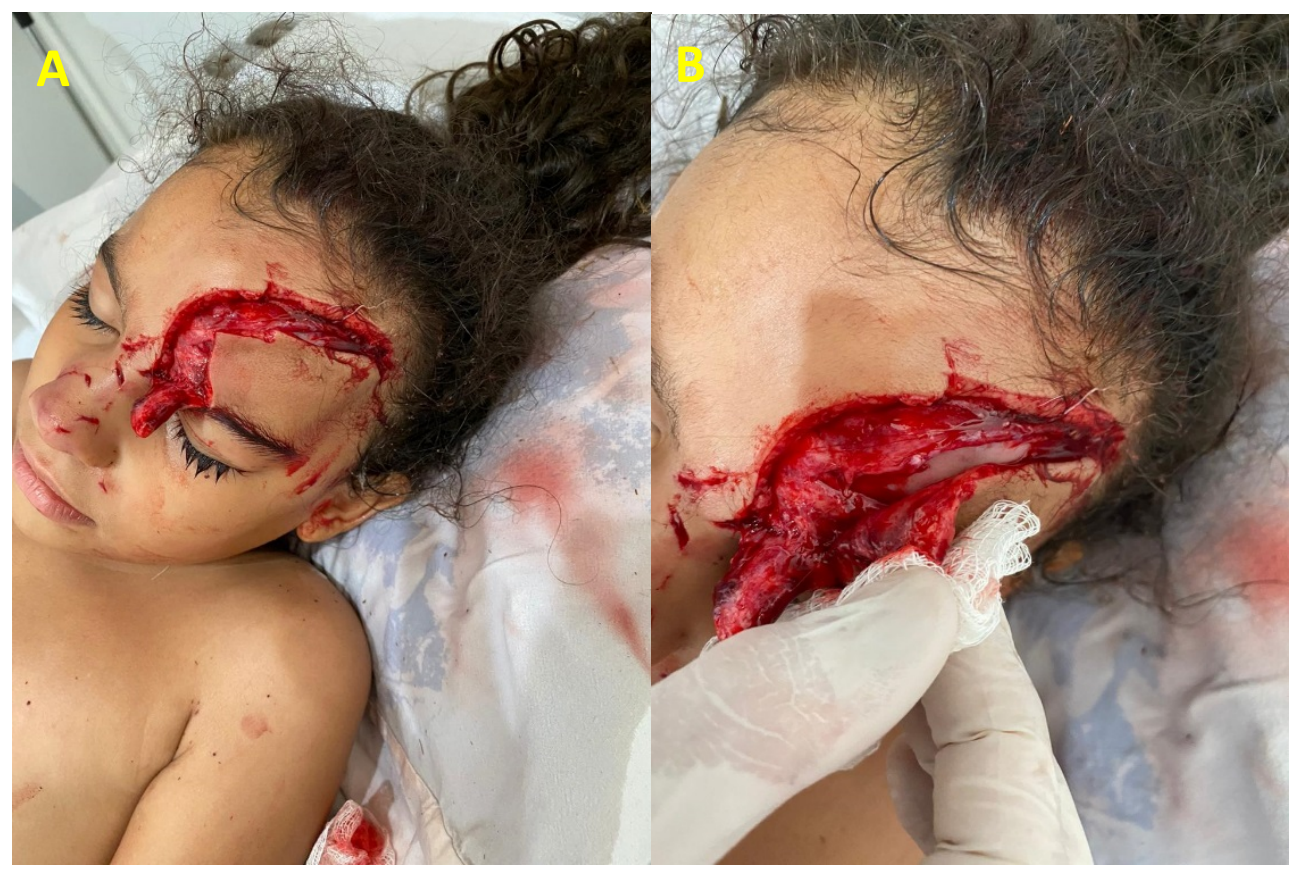

Fonte: Autores.

Após período de jejum, a paciente foi submetida a anestesia geral, para que os ferimentos pudessem ser tratados adequadamente. As feridas em face foram primeiramente limpas por meio de clorexidina degermante $4 \%$, seguido de uma antissepsia com clorexidina tópica 4\%. Foram realizados o debridamento e remoção de tecidos com coloração e aspectos alterados, seguido do reposicionamento dos tecidos moles a fim de recompor a anatomia e a estética da mesma. (Macedo et al.,2006; Macedo et al., 2004).

As suturas internas foram feitas com fio de ploglactina 910, (5.0) e as suturas em pele com nylon (6.0) (figura 2A). Foram feitos curativos compressivos para evitar a formação de hematomas. A paciente foi medicada com cefatriaxona, profenid, dipirona e feito todo o protocolo para mordedura de animais (Figura 2. B. C). 
Figura 2. A. Suturas internas feitas com fio de ploglactina 910, (5.0). B.. Sutura em pele para fechamento da lesão com nyloB(6.0). C. Paciente em pós operatório sob os devidos cuidados.

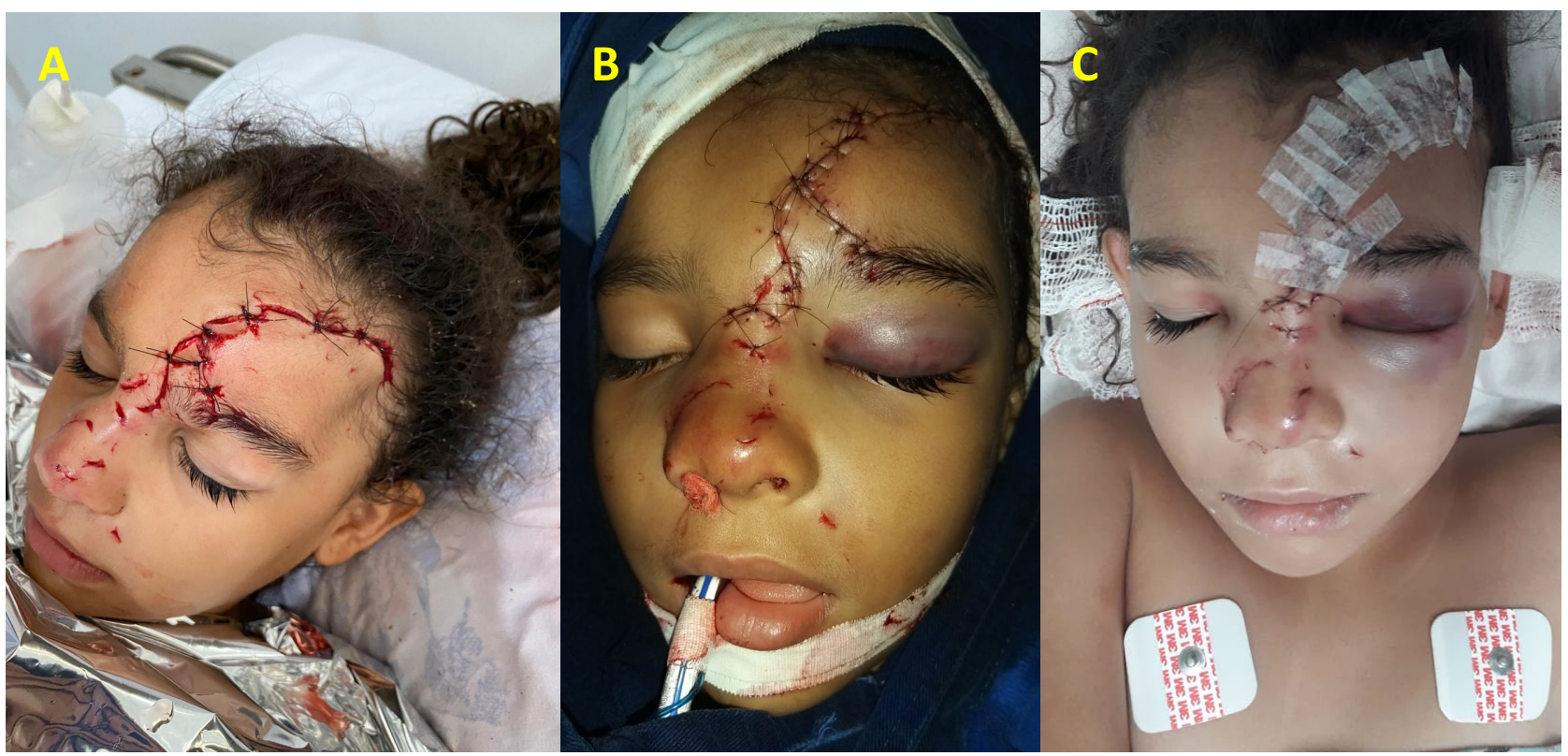

Fonte: Autores.

As suturas foram removidas em 7 dias, e recomendações quanto ao uso de pomada a base neomicina, filtro solar e evitar a exposição ao sol. Foram feitos acompanhamentos periódicos, analisando-se a cicatrização da ferida, como vemos nas Figuras (3. A. B).

Figura 3. A Pós operatório de 15 dias com uso de de pomada a base de neomicina. B. Pós operatório de 17 dias.

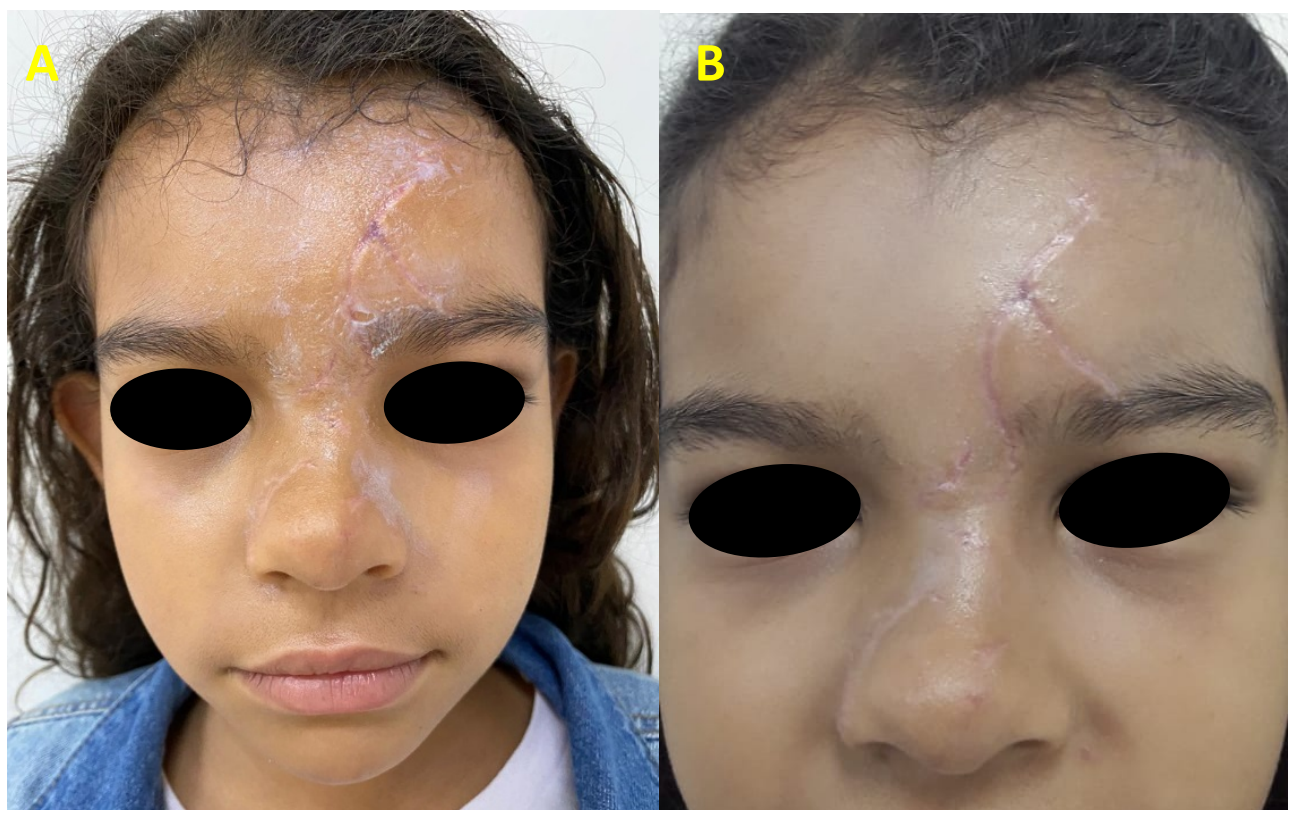

Fonte: Autores.

Também foi realizada terapia com laser para auxiliar o processo de cicatrização. A terapia com lazer consiste na aplicação de luz, promovendo reparação tecidual, diminuindo a inflamação e produzindo analgesia usando-se uma fonte de 
baixa potência. Foi utilizado um protocolo no qual foi feita a aplicação de azul de metileno, no local da lesão, $1 \mathrm{ml}$ para cada região de aplicação por aproximadamente 1 minuto e em seguida faz se a aplicação do laser de baixa potência com comprimento de onda de luz visível (660 Nanómetros) e energia de 6 joules por ponto de aplicação, durante 14 dias pósoperatório.

Há 3 meses do procedimento cirúrgico, a área operada ainda estava em fase de cicatrização (figuras 4. A e B). Foi feito acompanhamento de 8 meses para avaliação das cicatrizes em face, que ocorreram de forma satisfatória sem a formação de queloides (Figura 4. C).

Figura 4. A Pós operatório de 3 meses B.Lésão em processo de cicatrização. C. Pós operatódio de 8 meses com cicatrização completa da lesão.
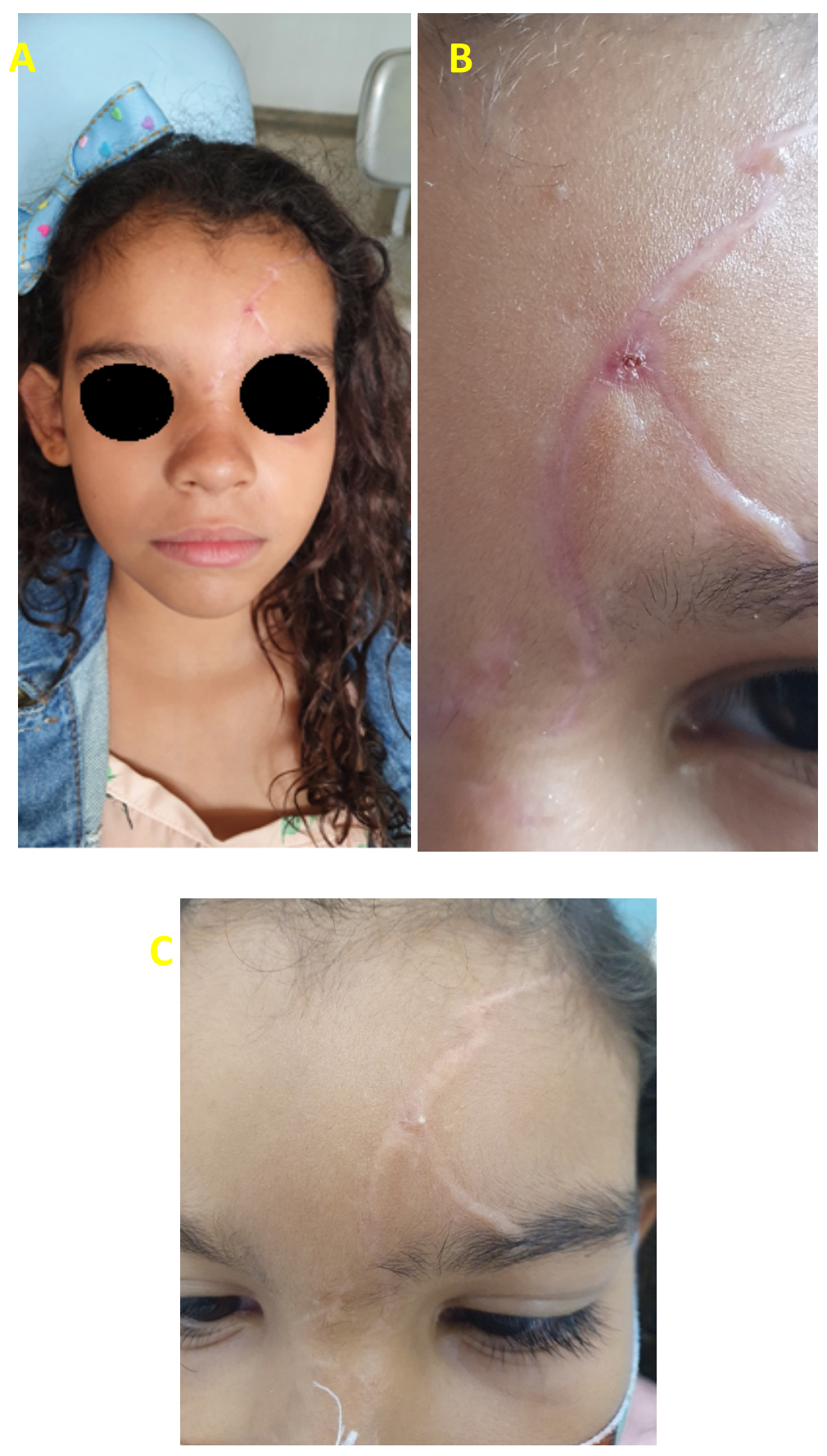

Fonte: Autores. 
Estudos mostram que a reparação cirúrgica imediata apresenta vantagens sobre o fechamento tardio pois lesões abertas demandam curativos diários, além de altas doses de analgésicos durante e após a troca destes. A sutura primária também permite ao cirurgião resolver o problema estético (Macedo et al.,2004).

Entretanto, para que haja uma boa cicatrização, é necessária uma coordenada e perfeita cascata de eventos celulares e moleculares que vão interagir para que ocorra a reconstituição e repavimentação do tecido (Ortonne et al., 1994). Tal evento é um processo dinâmico que envolve fenômenos bioquímicos e fisiológicos comportando-se de forma harmoniosa e garantindo a restauração tissular. Alguns autores consideram três estágios no processo de cicatrização: inicialmente um estágio inflamatório, seguido pelo de proliferação e finalizando com o reparo em um estágio de remodelação (Ortonne et al.,1994). Outros autores classificam de uma forma mais completa dividindo o processo em cinco fases principais como coagulação, inflamação, proliferação, contração da ferida e remodelação (Fazio et al.,2000).

A intervenção precoce no tratamento da ferida cirúrgica por meio da aplicação do laser de baixa intensidade pode ser uma importante estratégia terapêutica na aceleração da cascata de eventos celulares e moleculares atuando mais rapidamente no processo de cicatrização, profilaxia da infecção e redução de complicações (Allendorf et al.,1997; Almeida et al.,2006). Trata-se de uma terapia não invasiva, não térmica, asséptica, indolor, e sem efeitos colaterais (Leal et al., 2012). Diversos estudos demonstraram que tal terapia atua acelerando o processo de reparo tecidual, com efeitos analgésicos, anti-inflamatórios e de regeneração tecidual (Kitchen et al., 1991; Bjordal et al., 2006; Damante et al., 2008; Fulop et al., 2009; Henriques et al., 2010;), apresentan resultados satisfatórios na cicatrização de feridas (Santos et al., 2011a) como podemos ver, no caso relatado no qual, em apenas 3 meses já havia um reparo quase que total da lesão e com 8 meses o reparo completo, satisfatório e sem presença de queloide.

\section{Discussão}

Lesões ocasionadas por mordidas de animais, representam grande parte das agressões físicas observadas nas emergências de hospitais, com ênfase para a mordedura de cães e gatos domésticos, principais envolvidos nesses tipos de situações, vista a constante exposição das vítimas ao agente agressor (Santos et al.,2007; Abuabara et al.,2006).

Tristemente, as crianças são as mais acometidas, segundo o CDC de Atlanta pois $26 \%$ das mordeduras em crianças necessitam de cuidados médicos, comparado com 12\% em adultos, e as crianças são as principais vítimas fatais dos ataques caninos (Sacks. Et al., 1996). Por serem menores, elas sofrem mordeduras na cabeça e tem lesões mais desfigurantes que os adultos. Entre as crianças com menos de 9 anos, 73\% das lesões são na cabeça, enquanto nas outras idades, somente 30\% das lesões ocorrem neste segmento do corpo (Weiss et al.,1998).

O risco de infecções é uma das principais preocupações inerentes aos ferimentos por mordeduras e deve-se estabelecer uma abordagem imediata de profilaxia, como limpeza local e irrigação abundante dos ferimentos com soro fisiológico, visando sua prevenção. Em ferimentos penetrantes profundos, a irrigação procede-se com auxílio de seringa e agulha (Kesting et al.,2006; Mathur et al.,2011; Stefanopoulos et al.,2005; Rui-Feng et al.,2013). Portanto, é essencial a realização de um exame clínico completo, associado a uma exploração detalhada da ferida sob anestesia geral conforme o caso.

Em casos mais severos, completando a conduta terapêutica, é desejável encaminhar a vítima da mordedura para um acompanhamento psicológico. Esses pacientes podem manifestar dificuldades de ajustamento social após o trauma, principalmente quando apresentam lesões desfigurantes (Rusch et al.,2000). 


\section{Considerações Finais}

Não foram identificadas complicações infecciosas no paciente relatado. O fechamento imediato das mordeduras caninas ou humanas na face e no couro cabeludo é seguro, até em casos após várias horas da lesão (Fleisher et al., 1999; Tu et al., 2002; Macedo et al.,2004).

É necessário reforçar que uma boa anamnese e manejo clínico inicial do paciente são imprescindíveis ao sucesso do tratamento, bem como de uma antissepsia, profilaxia antibiótica, desbridamento e suturas imediatas. Segundo a literatura, a profilaxia antirrábica é preconizada para mordeduras causadas por animais domésticos de que não se conhece a história de imunização e quando indicados, devem sempre fazer parte do protocolo de tratamento desses pacientes (Macedo et al.,2006; Porto et al.,2016; Santos et al.,2007).

\section{Referências}

Abuabara A. (2006). A review of facial injuries due to dog bites. Med Oral Patol Oral Cir Bucal. 11:E348-50.

Allendorf, J. P., Bessler, M., Huang, J., Kayton, M. L. \& Laird, D. (1999). Heliumneon laser irradiation at flences of 1,2 and 4 J/cm 2 failed to accelerate wound healing as assessed by wound contracture rate and tensile strength. Lasers in Surgery and Medicine, v.20, n.3, p.340-345.

Almeida, R. M (2006). Avaliação do processo de cicatrização de lesões, tratadas com laser de baixa intensidade, através de sistema de aquisição e tratamento de imagem. Dissertação de Mestrado, Programa de Pós Graduação Escola de Engenharia Mecânica Universidade Federal de Minas Gerais.

Bagheri, S. C., Bell, R. B. \& Khan, H. A. (2013). Terapias Atuais em Cirurgia Bucomaxilofacial. Rio de Janeiro: Elsevier;

Bjordal, J.M., Johnson, M. I., Iversen, V., Aimbire, F. \& Lopes-Martins, R. V. B. (2006). Low-level laser therapy in acute pain: A systematic review of possible mechanisms of action and clinical effects in randomized placebo-controlled trials. Photomedicine and Laser Surgery, v.24, n.2, p.158-168.

Damante, C. A., Marques, M. M \& De Micheli, G. (2008). Terapia com laser em baixa intensidade na cicatrização de feridas: revisão de literatura. Revista da Faculdade de Odontologia Universidade de passo Fundo, v.13, n.3, p.88-93.

Estrela, C. (2018). Metodologia Científica: Ciência, Ensino, Pesquisa. Editora Artes Médicas.

Fazio, M. J., Zitelli, J. Á. \& Goslen, J. B. (2000). Cicatrização de feridas. In: Coleman III WP, Hanke CW, Alt TH, Asken S. Cirurgia Cosmética - Principios e Técnicas. 2.ed. Rio de Janeiro: Revinter 23-28.

Fleisher, G. R. (1999). The management of bite wounds. N Engl J Med.340(2):138-40.

Fulop, A.M., Dhimmer, S., Deluca, J. R., Johanson, D. D., Lenz, R. V., Patel, K. B., Douris, P. C \& Enwemeka, C.S. (2009). A meta-analysis of the efficacy of phototherapy in tissue repair. Photomedicine and Laser Surgery, v.27, n.5, p.695-702.

Henriques, A. C., Cazal, C. \& Castro, J. L. (2010), Ação da laserterapia no processo de proliferação e diferenciação cellular: revisão da literatura. Revista do Colégio Brasileiro de Cirurgiões, v.37, n.4, p.295-302.

Kitchen, S. S. \& Partridge, C. J. (1991). A review of low level laser therapy. Journal of Physiotherapy, v.77, n.3, p.161-168.

Kesting, M. R., Hölzle, F., Pox, C., Thurmüller, P. \& Wolff, K. D. (2006). Animal bite injuries to the head: 132 cases. British Journal of Oral and Maxillofacial Surgery. 44: 235-239.

Leal, C.T., Bezerra, A.L \& Lemos, A. (2012). A efetividade do laser de HeNe 632,8 nm no reestabelecimento da integridade dos tecidos cutâneos em animais experimentais: revisão sistemática. Revista Fisioterapia e Pesquisa, v.19, n.3, p.290-296.

Macedo, J. L. S. \& Rosa, S. C. (2004). Reconstrução de couro cabeludo após mordedura canina. Rev Col Bras Cir.31(1):27-33.

Macedo, J. L.S., Camargo, L. M. \& Almeida, P. F. (2006). Estudo prospectivo do fechamento primário das mordeduras caninas e humanas na face e no couro cabeludo. Rev. Soc. Bras. Cir. Plást. 21(1): 23-9.

Mathur A., Ramesh, K. \& Kumar, G. A. (2011). Management of animal bite wounds on face: our experience. World Journal of Dentistry. $2(4)$ :309-311.

Miloro, M., Ghali, G. E., Larsen, P. E. \& Waite, P. D. (2013). Princípios de Cirurgia Bucomaxilofacial de Peterson. 2.ed. São Paulo: Santos.

Ortonne, J. P., Clévy, J. P. Physiologie de la cicatrisation cutanée. Ver Prat 1994; 44(13): 1733-4.

Ortonne, J. P. \& Clévy, J. P. (1944). Physiologie de la cicatrisation cutanée. Rev. Prat 44(13); 1735-7.

Pereira, A. S., Shitsuka, D. M., Parreira, F. J., \& Shitsuka, R. (2018). Metodologia da Pesquisa Científica (1a ed. E-book). Santa Maria: UAB/NTE/UFSM.

Porto, G. G., Souza, B. L. M. \& Sampaio, D. O. (2013). Manejo de lesões por mordedura de animal: relato de casos. Rev. Cir. Traumatol. Buco- MaxiloFac., Camaragibe. 13 (4): 39-44. 
Research, Society and Development, v. 10, n. 7, e18410716463, 2021

(CC BY 4.0) | ISSN 2525-3409 | DOI: http://dx.doi.org/10.33448/rsd-v10i7.16463

Rui-Feng, C., Li-Song, H, Ji-Bo, Z. \& Li-Qiu, W. (200). Emergency treatment on facial laceration of dog bite wounds with immediate primary closure: a prospective randomized trial study. BMC Emergency Medicine 2013, 13(Suppl 1):S2.

Rusch, M. D., Grunert, B. K., Sanger, J. R, et al. - Psychological adjustment in children after traumatic disfiguring injuries: a 12-month follow-up. Plast Reconstr Surg. 106(7):1451-1460.

Sacks, J. J., Lockwood, R. \& Hornreich, J, et al. (1989-1994). Fatal dog attacks., (1996-1997). Pediatrics, (6 pt 1): $891-895$.

Santos, N. R. S., Sobrinho, J. B. M., Almeida, P. F., Ribeiro, A. A., Cangussú, M. C. T., Santos, J. N \& Pinheiro, A. L. B. (2011a) Influence of the combination of infrared and red laser light on the healing of cutaneous wounds infected by staphylococcus aureus. Photomedicine and Laser Surgery, v.29, n.3, p.177-182.

Santos, T. S., Antunes, A. A., Carvalho, R. W. F., Avelar, R. L., Melo, R. \& Dourado, E. (2007). Perfil das pacientes vitimas de mordeduras faciais: um estudo retrospectivo. RGO, Porto Alegre. 2007; v. 55, 4: 369-373.

Stefanopoulos, P. K. \& Tarantzopoulou, A. D. (2005). Facial bitewounds: management update. Int. J. Oral Maxillofac.Surg. 34: 464-472.

Tu, A. H., Girotto, J. A., Singh, N., Dufresne, C, R., Robertson, B. C. \& Seyfer, A. E. et al. (2002). Facial fractures from dog bite injuries. Plast Reconstr Surg.109(4):1259-65.

Weiss, H.B., Friedman, D. I. \& Coben, J. H . (1998). Incidence of dog bite injuries treated in emergency departments. JAMA, $1998,279(1): 51-53$. 